\title{
Two Regimes of Slow-Light Losses Revealed by Adiabatic Reduction of Group Velocity
}

\author{
R. J. P. Engelen, ${ }^{1}$ D. Mori, ${ }^{2}$ T. Baba, ${ }^{2}$ and L. Kuipers ${ }^{1}$ \\ ${ }^{1}$ Center for Nanophotonics, FOM Institute for Atomic and Molecular Physics (AMOLF), \\ Kruislaan 407, 1098 SJ Amsterdam, The Netherlands \\ ${ }^{2}$ Department of Electrical and Computer Engineering, Yokohama National University, \\ 79-5 Tokiwadai, Hodogayaku, Yokohama 240-8501, Japan
}

(Received 2 April 2008; revised manuscript received 3 July 2008; published 3 September 2008)

\begin{abstract}
The losses in a photonic crystal waveguide were measured with a near-field microscope in the group velocity range of $c / 7$ down to $c / 200$. Our measurements show that the losses scale proportional to $v_{g}^{-2}$ for group velocities above $c / 30$. Below $c / 30$, the losses are no longer described by the same power-law dependence on $v_{g}$ and the modal pattern becomes irregular, indicative of multiple scattering. The findings indicate the existence of two regimes of slow-light losses: one where a perturbative approach describes propagation with fabrication disorder and one where it breaks down.
\end{abstract}

DOI: 10.1103/PhysRevLett.101.103901

PACS numbers: 42.70.Qs, 42.25.Fx, 42.82.Et, 68.37.Uv

Photonic crystals (PhCs) consist of a periodic arrangement of dielectric materials, usually with a high refractive index contrast. In a $\mathrm{PhC}$ with properly chosen geometry and materials, light can be strongly influenced by the periodic structure. Numerous interesting properties have been reported over the years, like photonic band gaps [1], negative refraction [2], and superlensing [3]. One of the intriguing properties of $\mathrm{PhCs}$ is that light can be made to propagate with low group velocities at specific optical frequencies [4,5]. This "slow light" can enhance nonlinear effects [6], useful for future on-chip all-optical switching.

In two-dimensional photonic crystal waveguides, light can in theory propagate without losses [7]. However, unavoidable structural imperfections like variations in hole diameter or shape, roughness of the interfaces of the structure, and/or slight displacement of the holes will result in scattering out of the waveguide mode, thus inducing loss. We will refer to these imperfections as the disorder in the structure. The major loss channels resulting from the disorder are out-of-plane radiation losses and scattering in the backward direction in the waveguide. Since slow-light propagation is intrinsically linked to a very strong interaction with the lattice in which the waveguide is embedded, the losses per unit length increase with decreasing group velocity [8]. Although the disorder created by the structural imperfections is disadvantageous for efficient guiding of light, it can give rise to interesting optical transport phenomena involving multiple scattering $[9,10]$.

There has been significant effort to determine how the losses in photonic crystal waveguides ( $\mathrm{PhCWs}$ ) scale with the group velocity $\left(v_{g}\right)$. Hughes et al. [8] suggested in a theoretical study that the total losses scale proportional to $v_{g}^{-2}$. Crucial to this study and alike $[8,11]$, is that the disorder is considered to be a small perturbation on the geometry of the waveguide. The disorder therefore hardly affects the propagation of light apart from the losses scattered out of the mode. There have been a few studies that attempted to elucidate the scaling of the losses experimentally [12-14]. In these studies several waveguides with different lengths were fabricated. By comparing the transmission spectra of the structures for the different lengths, the losses may be determined. For such a study it is crucial that only the waveguide length differs and the mean optical properties are exactly equal. The reported results in Refs. [12-14] range between a proportionality of the losses with $v_{g}^{-1 / 2}$ and $v_{g}^{-2}$, corresponding to loss exponents $(\alpha)$ of 0.5 and 2 , respectively.

Here we investigate the propagation of light and the losses directly by monitoring the optical field in a PhCW en route, with a phase-sensitive near-field microscope. The waveguide under investigation is a so-called chirped waveguide: the hole radius is gradually increased along the propagation direction. As a result, the light slows down adiabatically, which leads to a position-dependent group velocity. By changing the optical frequency, the position in the waveguide for which a specific group velocity occurs can be controlled, with each position having its own local realization of unintentional disorder. We show by visualization of the modal pattern as well as a quantitative analysis of the losses averaged over many realizations of the disorder that a perturbative approach to dealing with disorder breaks down at low group velocities, in our case below $c / 30$, as in this slow-light regime multiple scattering becomes important.

Figure 1 shows a schematic of the waveguide under investigation. A PhCW is created by removing a single row of holes in the photonic crystal lattice $[15,16]$. From left to right the hole radius is linearly increased, resulting in a chirped $\mathrm{PhCW}$ [17]. We used an air-bridge structure with a $210 \mathrm{~nm}$ thick silicon membrane with a hexagonal lattice of air holes with a $456 \mathrm{~nm}$ period $(a)$. The hole radius increases linearly from 142 to $150 \mathrm{~nm}$ over a length of 300 lattice periods, with typical local fabrication variations in diameter of approximately $3 \mathrm{~nm}$. A portion of the 

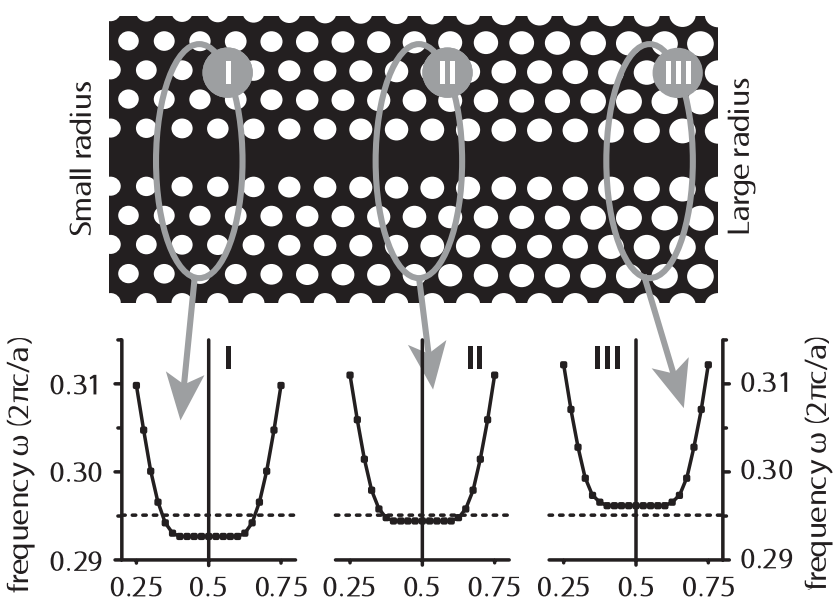

wave vector $k$ (units $2 \pi / a)$

FIG. 1. Schematic representation of the geometry of the chirped waveguide (top). The radius of the holes in the $\mathrm{PhC}$ lattice is linearly increased from left to right. The waveguide mode (continuous line) in the dispersion relation shifts up as the hole size increases. Light with a specific optical frequency (dotted line) is therefore allowed to propagate in regions I (with a relatively high $v_{g}$ ) and II (lower $v_{g}$ ), but not in region III. The dispersion relations in regions I and III are calculated by 3D FDTD for hole radii of $0.306 a$ and $0.321 a$, respectively. For all other radii, they are obtained by linear interpolation (with an error of maximally $0.03 \%$ in frequency).

dispersion relation calculated by $3 \mathrm{D}$ finite-difference timedomain (FDTD) calculations is shown in Fig. 1, for different sections of the $\mathrm{PhCW}$. In these calculations each section is treated as if it were infinitely long, i.e., with a constant hole radius. In the dispersion relation, the waveguide mode flattens as the wave vector approaches the Brillouin zone boundary at $k=0.5$ (in units of $2 \pi / a$ ), at which the group velocity ( $v_{g} \equiv d \omega / d k$ ) is zero.

As the hole size increases, the waveguide mode in the dispersion relation shifts to higher frequencies. For light with a certain optical frequency (dashed line in Fig. 1), this leads to a reduction of the group velocity along the propagation direction until the waveguide no longer allows propagation (section III). We will refer to the region where light is propagating with $v_{g}<c / 30$ as the cutoff region. This region corresponds to group indices (defined as $n_{g}=$ $c / v_{g}$ ) above 30. After this region, the light must either have been reflected back towards the input or have been scattered out of plane.

The optical field inside the waveguide was measured using a phase-sensitive near-field microscope [18]. In this microscope, we scan a near-field probe over the sample at approximately $10 \mathrm{~nm}$ height. A small portion of the optical field in the sample couples to the probe [19]. Variations in height during scanning $( \pm 3 \mathrm{~nm}$ ) will result in variations in coupling efficiency of at most $5 \%$. By scanning the probe over the surface we can map the electric ( $E$ field) distribution in the waveguide. Figure 2(a) shows the amplitude of the $E$ field picked up in a near-field measurement at $\omega=0.2961$. The waveguide is oriented horizontally, centered around $y=2.8 a$, and the light is incident from the left. We observe that the modal pattern does not change dramatically up to $x=190 a$. In the cutoff region (190a< $x<230 a$ ), the pattern broadens laterally and becomes irregular along the propagation direction.

With our near-field microscope we also recovered the phase of the propagating light. By analyzing the phase evolution over 9 lattice periods, the dominant or local wave vector was determined. For the measurement at $\omega=0.2961$, the local wave vector is plotted versus position in Fig. 2(b). The expected local wave vector, based on the theoretical dispersion relation, is depicted in red in Fig. 2(b). The results for 25 measurements, each at a different optical frequency, all show very good correspondence with the results obtained from the calculated dispersion relation. By exploiting the correspondence between

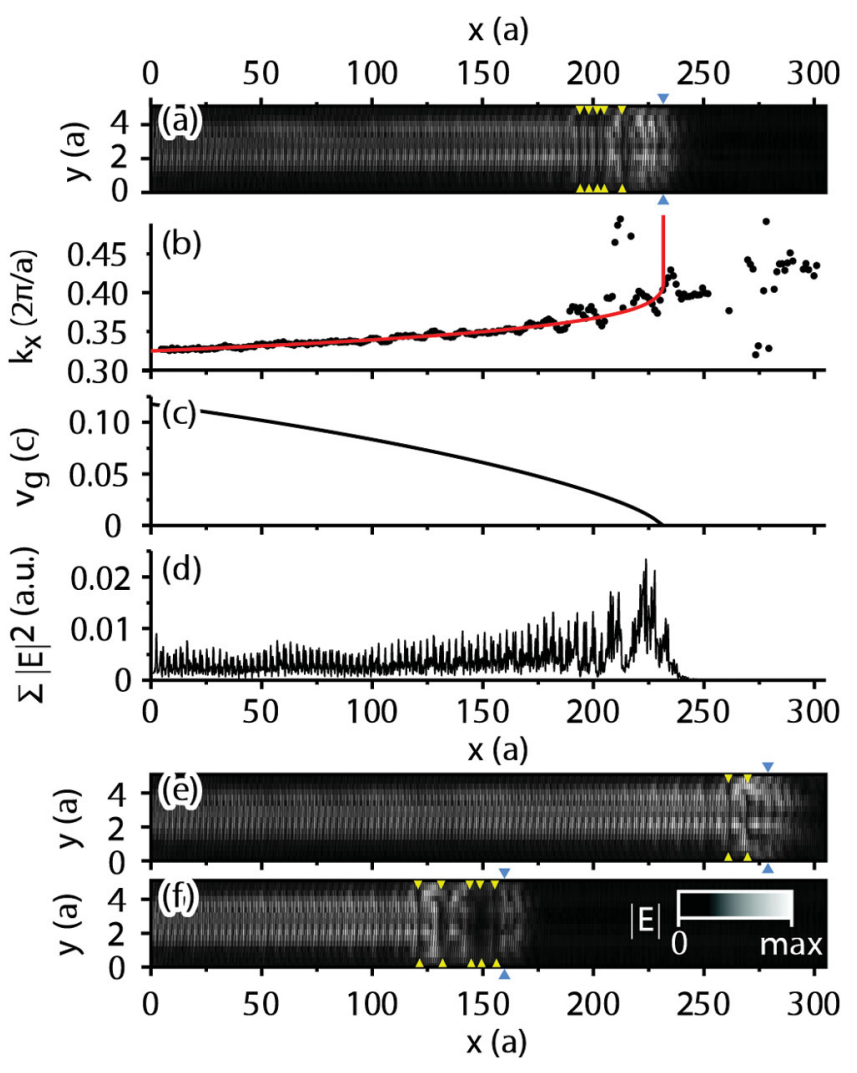

FIG. 2 (color). (a) E-field amplitude from a near-field measurement on the chirped waveguide at $\omega=0.2961$ (in units $2 \pi c / a$ ). (b) Local wave vector as a function of $x$ position, obtained from the measurement in (a) (solid points) The solid (red) line depicts the expected wave vector calculated with the dispersion relations of Fig. 1. (c) Group velocity corresponding to the calculated data in (b). (d) Summed intensity $\left(|E|^{2}\right)$ over all $y$ points of the image in (a), yielding the total intensity in the waveguide as a function of position $x$. (e),(f) Measured $E$-field amplitude of the same waveguide and area as in (a), now at $\omega=$ 0.2973 and 0.2950 , respectively. The yellow triangles in (a),(e), (f) indicate local minima. The blue triangles indicate the expected cutoff position. 
experiment and calculations, we determined the group velocity $v_{g}$ as a function of position $x$ from the calculated data. The result is depicted in Fig. 2(c) and shows that the group velocity is $0.12 c$ at $x=0$ and gradually decreases, until it reaches zero at $x=230$.

In order to analyze the experimental data, we summed the intensity $\left(|E|^{2}\right)$ of the measurement in Fig. 2(a) along the $y$ direction in order to suppress the effect of mode pattern differences so that the energy of the mode can be determined as a function of position along the propagation direction. The thus obtained energy as a function of position is depicted in Fig. 2(d). The energy has a short-range oscillation due to two effects: the Bloch wave character of the guided mode [20] and interference of forward propagating and reflected light. Without out-of-plane losses the amplitude of the two is equal. Because of out-of-plane losses, that occur primarily in the cutoff region, the amplitudes may differ. The detected intensity shows dramatic changes in the cutoff region $(190 a<x<230 a)$ : the intensity increases and becomes irregular. We have highlighted local minima in the cutoff region with yellow triangles in Figs. 2(a), 2(e), and 2(f). At $x=230$, highlighted with the blue triangles, the group velocity is zero.

When we repeat the experiment at different optical frequencies, we observe qualitatively the same results: a gradual increase of the energy at larger propagation distances and an irregular pattern for positions where $v_{g}<$ $c / 30$. The first, expected difference is a shift in position with respect to the measurement at $\omega=0.2961$, as can be seen in Figs. 2(e) and 2(f) for $\omega=0.2973$ and $\omega=$ 0.2950 , respectively. Note that these are measurements on the same waveguide and the same measurement area. The position where light stops propagating is clearly dependent on frequency. For all 25 optical frequencies used in this investigation, we observe that the mode pattern becomes irregular in the cutoff region [19]. The pattern is different for each frequency, despite the small increments in frequency $(\Delta \omega<0.07 \%)$ used. The irregularities in the mode patterns are attributed to disorder. The fact that the pattern of the irregularity changes dramatically when a different local realization of the disorder is probed with the same low group velocity, e.g., compare Figs. 2(a) and 2(f), is a fingerprint for multiple scattering [21].

In order to properly quantify the relation between group velocity and losses, one needs to average over the many realizations of the local disorder underlying the losses. This necessity becomes particularly clear considering the large irregularities observed in the cutoff region. We therefore averaged our analysis over 25 measurements, with different optical frequencies, where the relation of group velocity and position is the same for each frequency, apart from a shift in position. By averaging over many frequencies, we average over different realizations of the disorder within a single sample, while the coupling of light into the waveguide remains unchanged.

Figure 3 depicts the intensity picked up by the probe $\left(I_{\text {probe }}\right)$ as a function of the group index, averaged over 25 effective realizations. Before averaging, the intensity of each measurement is normalized to the intensity at a velocity of $c / 20$, to account for possible differences in coupling efficiency to the waveguide. Figure 3 shows that the detected intensity increases sublinearly with group index between $n_{g}=7$ and $n_{g}=30$. When the group index increases further, the probed intensity levels off and remains between $I_{\text {probe }}=1.0$ and 1.5. Despite the averaging over 25 measurements, the intensity still varies in the cutoff region $\left(n_{g}>30\right)$ due to the irregular patterns of the individual measurements.

We attribute the increase of the intensity between $n_{g}=$ 7 and 30 in Fig. 3 to an increase of the $E$-field amplitude in the waveguide associated with the reduced group velocity. The intensity $\left(|E|^{2}\right)$ of light in photonic crystal waveguides is expected to be proportional to the group index [6]. To the best of our knowledge, our measurement is the first direct observation that the local energy density increases in a photonic crystal as the group velocity decreases.

From these measurements, we can recover the losses in the waveguide as a function of group velocity. We can describe the intensity in the waveguide according to Lambert-Beer's law:

$$
I(x+d x)=I(x) e^{-A n_{g}^{\alpha}(x) d x},
$$

describing that an infinitesimal increase in position is accompanied by a loss which scales with $n_{g}^{\alpha}$, with $n_{g}$ a function of $x$. We keep the loss exponent $\alpha$ constant for all $n_{g}$, in order to compare our quantitative results with that of others. Using the assumption that $\left|n_{g}^{\alpha}(x) d x\right| \ll 1$, the

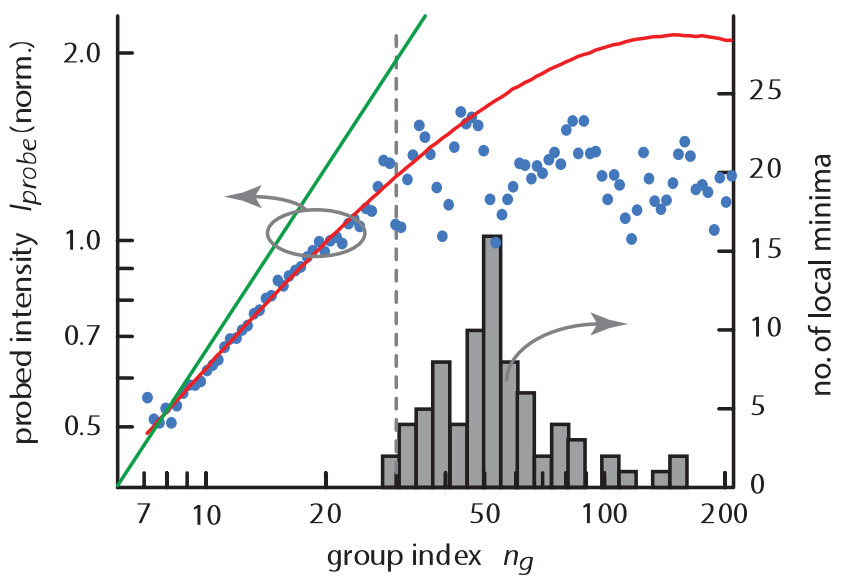

FIG. 3 (color). Intensity detected by the near-field probe as a function of the group index $n_{g}$, normalized at $n_{g}=20$. The data are averaged over 25 measurements, ranging between $\omega=$ 0.2936 and $\omega=0.2982$. The intensity of each individual measurement is normalized to $n_{g}=20$. The black line shows the expected result without losses. The red line represents the best fit to the measurements for the data points with $n_{g}<30$ only resulting in $\alpha=2$. The histogram depicts at what $n_{g}$ local intensity minima were observed, indicative of multiple scattering. 
above equation leads to

$$
I(x)=I(0) \exp \left[-A \int_{0}^{x} n_{g}^{\alpha}\left(x^{\prime}\right) d x^{\prime}\right],
$$

with $A$ being a loss coefficient, $I(0)$ the intensity input in the waveguide. When we want to fit this relation to the measured results, the integral needs to be evaluated, but since $n_{g}(x)$ cannot be approximated with a low-order polynomial, we have evaluated the integral in Eq. (2) numerically.

In the fit of Eq. (2) to the measured position-dependent intensity, the fit parameters were the loss exponent $\alpha$, the loss coefficient $A$, and $I(0)$. We could not find a satisfactory fit to the measured data for all $n_{g}$ 's. However, for only $n_{g}<$ 30 , we do find a close agreement with the measured results using $\alpha=2$. From the fit result, the loss at $n_{g}=5$ would be $2.4 \mathrm{~dB} / \mathrm{mm}$, which is a typical loss figure for these waveguides $[12,13]$.

The losses cannot be described by a single power of $n_{g}$, as is illustrated by the large discrepancy between the red curve and the measured data for $n_{g}>30$. In Fig. 3, we have also depicted a histogram of the occurrence of amplitude minima in the near-field measurements as a function of group index in an attempt to quantify where the irregular modal patterns occur. The irregular patterns appear at group indices above $n_{g}=30$ and most minima can be found around $n_{g}=50$. The occurrences decrease for higher $n_{g}$ as these $n_{g}$ 's are sampled less in the chirped waveguide. Strikingly, the group velocity at which the fitted red curve no longer shows agreement with the measured results coincides with the first appearance of local minima in the measurements.

The large discrepancy between theoretical predictions and the experimental observations can be explained as follows. The theoretical predictions are based on perturbation theory, which does not include multiple scattering phenomena. The multiple scattering leads to a change in the propagation properties, for example, by the formation of highly localized resonances [see around $x=160 a$ in Fig. 2(f)]. We discriminate two regimes of slow-light propagation. In the first regime the waveguide modes are well behaved and can be described with existing theory. In the second regime, at lower $v_{g}$, multiple scattering becomes important. Characteristic for this region is the presence of maxima and minima in the local field distribution. The group velocity for which the transition between the two regimes occurs will depend on the quality of the fabrication: the higher the quality, the smaller the group velocity for which multiple scattering becomes important. In our investigation, this transition occurs at roughly $c / 30$.

In conclusion, our measurements on a chirped photonic crystal waveguide show that the detected intensity increases in a near-field microscope as the group velocity in the waveguide decreases. The increase is due to the increase of the electromagnetic energy density in the waveguide. As the light propagates further, the group velocity decreases further and the detected intensity levels off. In the area where the losses increase, the modal pattern breaks up and becomes irregular. We found that for group velocities above $c / 30$, the losses scale proportional to $v_{g}^{-2}$, which is in agreement with perturbation theory. At smaller group velocities, the losses differ significantly from expectations based on a perturbative treatment of the disorder. At group velocities where a perturbative treatment of the losses fails, we observe irregular modal patterns. From our observations we conclude that two regimes should be considered when describing the propagation of light in a photonic crystal waveguide. One regime, for relatively high group velocities, in which light propagation is well described by the usual perturbative methods and one regime, for smaller group velocities, where multiple scattering becomes important and influences the propagation properties.

The authors acknowledge Femius Koenderink, Bernard Kaas, Ad Lagendijk, Philippe Lalanne, and Thomas Krauss for enlightening discussions. This research is supported by NanoNed, a nanotechnology program of the Dutch Ministry of Economic Affairs, and this work is part of the research program of FOM, which is financially supported by NWO.

[1] K. Edagawa, S. Kanoko, and M. Notomi, Phys. Rev. Lett. 100, 013901 (2008).

[2] A. Berrier et al., Phys. Rev. Lett. 93, 073902 (2004).

[3] E. Cubukcu et al., Phys. Rev. Lett. 91, 207401 (2003).

[4] H. Gersen et al., Phys. Rev. Lett. 94, 073903 (2005).

[5] Y. A. Vlasov et al., Nature (London) 438, 65 (2005).

[6] M. Soljačić and J. D. Joannopoulos, Nature Mater. 3, 211 (2004).

[7] R. D. Meade et al., J. Appl. Phys. 75, 4753 (1994).

[8] S. Hughes et al., Phys. Rev. Lett. 94, 033903 (2005).

[9] J. Topolancik, B. Ilic, and F. Vollmer, Phys. Rev. Lett. 99, 253901 (2007).

[10] J. Bertolotti et al., Phys. Rev. Lett. 94, 113903 (2005).

[11] D. Gerace and L. C. Andreani, Opt. Lett. 29, 1897 (2004).

[12] E. Kuramochi et al., Phys. Rev. B 72, 161318 (2005).

[13] Y. Tanaka et al., Electron. Lett. 40, 174 (2004).

[14] L. O'Faolain et al., Opt. Express 15, 13129 (2007).

[15] M. Notomi et al., Phys. Rev. Lett. 87, 253902 (2001).

[16] Z. Y. Li and K. M. Ho, Phys. Rev. Lett. 92, 063904 (2004).

[17] T. Baba et al., IEEE J. Sel. Top. Quantum Electron. 10, 484 (2004).

[18] M. Sandtke et al., Rev. Sci. Instrum. 79, 013704 (2008).

[19] See EPAPS Document No. E-PRLTAO-101-020837 for additional information and field amplitude maps of all the 25 measurements. For more information on EPAPS, see http://www.aip.org/pubservs/epaps.html.

[20] H. Gersen et al., Phys. Rev. Lett. 94, 123901 (2005).

[21] M. Gurioli et al., Phys. Rev. Lett. 94, 183901 (2005). 